Irish Math. Soc. Bulletin

Number 81, Summer 2018, 23-30

ISSN 0791-5578

\title{
Pedro Nunes and the Retrogression of the Sun
}

\author{
PETER LYNCH
}

What has been will be again, what has been done will be done

again; there is nothing new under the sun. Ecclesiastes 1:9

\section{INTRODUCTION}

In northern latitudes we are used to the Sun rising in the East, following a smooth and even course through the southern sky and setting in the West. The idea that the compass bearing of the Sun might reverse seems fanciful. But that was precisely what Portuguese mathematician Pedro Nunes showed in 1537. Nunes made an amazing prediction: in certain circumstances, the shadow cast by the gnomon of a sun dial moves backwards.

Nunes' prediction was counter-intuitive. We are all familiar with the steady progress of the Sun across the sky and we expect the azimuthal angle or compass bearing to increase steadily. If the shadow on the sun dial moves backwards, the Sun must reverse direction or retrogress. Nunes' discovery came long before Newton or Galileo or Kepler, and Copernicus had not yet published his heliocentric theory. The retrogression had never been seen by anyone and it was a remarkable example of the power of mathematics to predict physical behaviour. Nunes himself had not seen the effect, nor had any of the tropical navigators or explorers whom he asked.

Nunes was aware of the link between solar regression and the biblical episode of the sun dial of Ahaz (Isaiah, 38:7-9). However, what he predicted was a natural phenomenon, requiring no miracle. It was several centuries before anyone claimed to have observed the reversal (Leitão, 2017). In a book published in Lisbon (Nunes, 1537), Nunes showed how, under certain circumstances, the azimuth of the Sun changes direction twice during the day, moving first forwards, then backwards and finally forwards again. To witness this, the observer

2010 Mathematics Subject Classification. 46A99, 10A10.

Key words and phrases. Truth, Heron.

Received on 0-0-0000; revised 0-0-0000. 
must be located at a latitude lower than that of the Sun, that is, in the tropics with the Sun closer to the pole. Nunes was completely confident about his prediction:

"This is something surprising but it cannot be denied because it is demonstrated with mathematical certainty and evidence." (Quoted from Leitão, 2017).

Leitão, who has made a detailed study of Nunes' works, reviewed the method used by him. While Nunes' arguments are mathematically sound, they are difficult to follow, so we will demonstrate the retrogression in a more transparent way below. But first, let us look at Pedro Nunes himself.

\section{Pedro Nunes (1502-1578)}

Pedro Nunes (also known as Petrus Nonius), a Portuguese cosmographer and one of the greatest mathematicians of his time, is best known for his contributions to navigation and to cartography. Nunes studied at the University of Salamanca in Spain, a university already 300 years old at that time. He returned to Lisbon and was later appointed Professor of Mathematics at the University of Coimbra. In 1533 he qualified as a doctor of medicine and in 1547 he was appointed Chief Royal Cosmographer.

Nunes had great skill in solving problems in spherical trigonometry. This enabled him to introduce improvements to the Ptolemaic system of astronomy, which was still current at that time (Copernicus did not publish his theory until just before his death in 1543). Nunes also worked on problems in mechanics.

Much of Nunes' research was in the area of navigation, a subject of great importance in Portugal during that period: sea trade was the main source of Portuguese wealth. Nunes understood how a ship sailing on a fixed compass bearing would not follow a great circle route but a spiraling course called a loxodrome or rhumb line that winds in decreasing loops towards the pole. Nunes taught navigation skills to some of the great Portuguese explorers. He has a place of prominence on the Monument to the Portuguese Discoveries in Lisbon, which shows several famous navigators (Figs. 1 and 2).

\section{AnAlysis of Solar Retrogression}

Nunes demonstrated the retrogression using spherical trigonometry. This was long before Newton's laws or differential calculus 


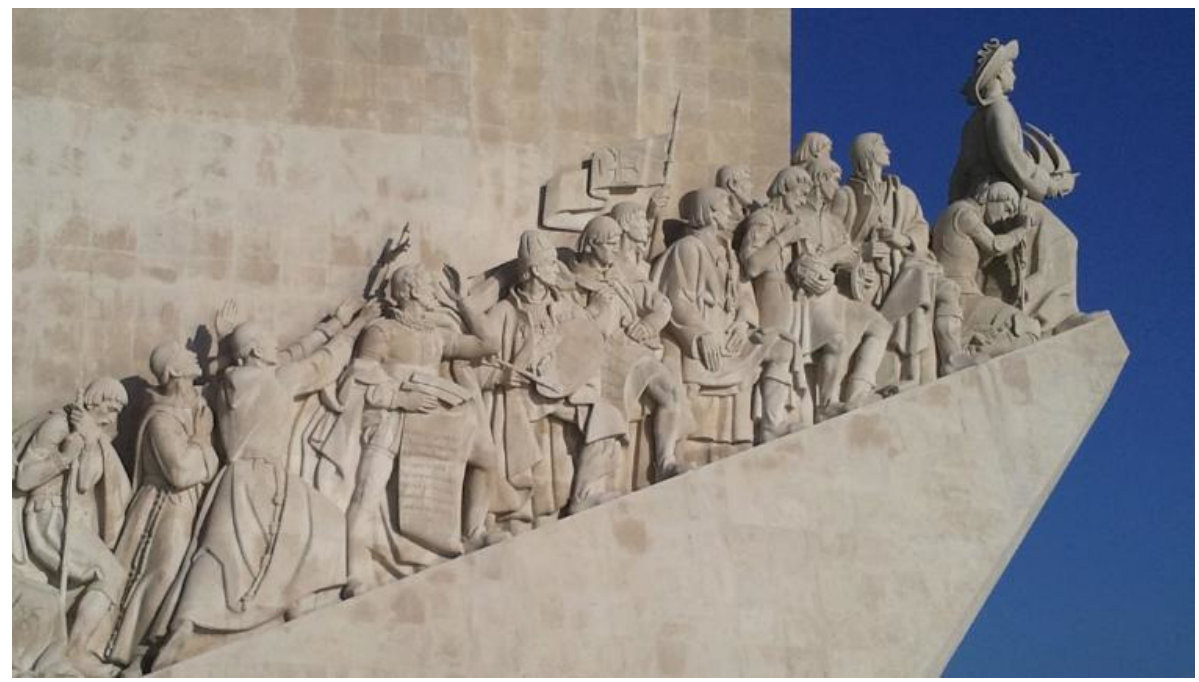

Figure 1. Monument to the Portuguese Discoveries, Lisbon.

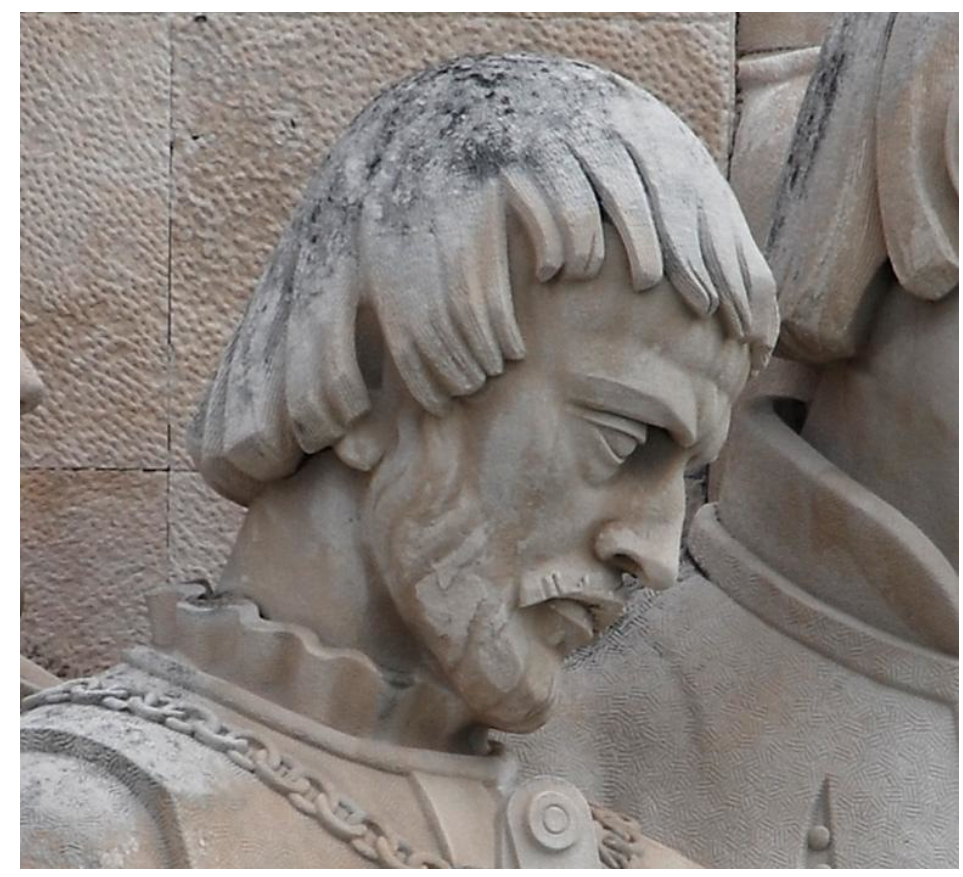

Figure 2. Pedro Nunes (1502-1578) (detail of Monument to Portuguese Discoveries, Lisbon).

were available. In this section we derive the condition for the phenomenon, using a simple transformation and elementary differential calculus. An expression is found for the azimuth of the Sun as a function of the time. For reversal to occur, the derivative of this function must vanish. The condition follows immediately from this. The result has been known for centuries (e.g., Morrison, 1898) but the derivation below is simpler than most previous accounts. 
Frames of Reference. We begin with a cartesian frame $(x, y, z)$ fixed relative to Earth and rotating with it. The origin is at the centre of the Earth and the $x$-axis passes through the point where the prime meridian intersects the equator. There is an associated polar coordinate frame $(r, \theta, \lambda)$ with colatitude $\theta$ and longitude $\lambda$. The latitude is $\phi=\frac{\pi}{2}-\theta$.

We assume that the Sun is at a fixed latitude $\phi_{S}$. If its longitude at Noon is $\lambda_{O}$, then its longitude at time $t$ is $\lambda_{S}=\lambda_{O}-\Omega\left(t-t_{O}\right)$ where $\Omega$ is the angular velocity of Earth. Given the distance $A$ from Earth to Sun, the cartesian coordinates of the Sun are

$$
\left(x_{S}, y_{S}, z_{S}\right)=\left(A \cos \lambda_{S} \cos \phi_{S}, A \sin \lambda_{S} \cos \phi_{S}, A \sin \phi_{S}\right) .
$$

The coordinates of the observation point $P_{O}$ are $\left(x_{O}, y_{O}, z_{O}\right)$ and from these the polar coordinates are easily found: $\left(a, \theta_{O}, \lambda_{O}\right)$ where $a$ is the Earth's radius. There is no loss of generality in assuming that the observatory is on the prime meridian. Then its latitude and longitude are $\left(\phi_{O}, \lambda_{O}\right)=\left(\frac{\pi}{2}-\theta_{O}, 0\right)$.

We define local cartesian coordinates $(X, Y, Z)$ at the observation point by rotating the $(x, y, z)$ frame about the $y$-axis through an angle equal to the colatitude $\theta_{O}$. The $Z$-axis then points vertically upward through $P_{O}$. Moving the origin to $P_{O}$, the $(X, Y)$ plane becomes tangent to the Earth at this point. The cartesian coordinates of the Sun in the new system are given by the affine transformation

$$
\left(\begin{array}{l}
X_{S} \\
Y_{S} \\
Z_{S}
\end{array}\right)=\left[\begin{array}{ccc}
\cos \theta_{O} & 0 & -\sin \theta_{O} \\
0 & 1 & 0 \\
\sin \theta_{O} & 0 & \cos \theta_{O}
\end{array}\right]\left(\begin{array}{l}
x_{S} \\
y_{S} \\
z_{S}
\end{array}\right)-\left(\begin{array}{l}
0 \\
0 \\
a
\end{array}\right) .
$$

In fact, since the distance to the Sun is enormous relative to the radius of the Earth, we can omit the last term $(0,0, a)^{\mathrm{T}}$ without significant error. Then, in terms of the latitude of the observation point, the solar coordinates are

$$
\left(\begin{array}{c}
X_{S} \\
Y_{S} \\
Z_{S}
\end{array}\right)=\left[\begin{array}{ccc}
\sin \phi_{O} & 0 & -\cos \phi_{O} \\
0 & 1 & 0 \\
\cos \phi_{O} & 0 & \sin \phi_{O}
\end{array}\right]\left(\begin{array}{l}
x_{S} \\
y_{S} \\
z_{S}
\end{array}\right) .
$$

The latitude and longitude of the Sun in the rotated system are

$$
\Phi_{S}=\arcsin \left[Z_{S} / A\right], \quad \Lambda_{S}=\arctan \left[Y_{S} / X_{S}\right]
$$

and the azimuth and elevation (or altitude) of the Sun are

$$
\alpha=\pi-\Lambda_{S}, \quad e=\Phi_{S} .
$$


Variation of the Azimuthal Angle. To demonstrate the circumstances in which retrogression of the Sun occurs, we take the time derivative of the azimuthal angle of the Sun. This is given by (4). We use (3) to express the Sun's latitude and longitude in the rotated cartesian frame and then the transformation (2) for the original cartesian coordinates. Finally, (1) gives an expression for the azimuth in terms of the variables $\left\{\lambda_{S}, \phi_{S}, \phi_{O}\right\}$. The two latitudes are fixed in time while the longitude $\lambda_{S}$ varies as $\lambda_{S}=-\Omega\left(t-t_{O}\right)$, where $t_{O}$ is the time at Noon.

If the Sun is to retrogress, the time derivative of the azimuth must vanish. We find that

$$
\tan \Lambda_{S}=\frac{Y_{S}}{X_{S}}=\frac{\sin \lambda_{S} \cos \phi_{S}}{\cos \lambda_{S} \cos \phi_{S} \sin \phi_{O}-\sin \phi_{S} \cos \phi_{O}}
$$

The vanishing of the derivative leads, after some manipulation, to the equation

$$
\cos \lambda_{S}=\frac{\tan \phi_{O}}{\tan \phi_{S}}
$$

Clearly, there will be an azimuth at which the derivative vanishes only if the right hand side is less than unity, that is, if

$$
\phi_{O}<\phi_{S} .
$$

This means that retrogression will be seen only if the observation point is between the Equator and the latitude of the Sun. In particular, it must be in the tropics. Eq. (5) corresponds to Eq. (5) in Morrison (1898).

Numerical Results. We consider the daily path of the Sun at the time of the Summer solstice $\left(\phi_{S}=23.5^{\circ} \mathrm{N}\right)$ for observations from an extra-tropical point $\left(\phi_{O}=40^{\circ} \mathrm{N}\right)$ and a point within the tropics $\left(\phi_{O}=20^{\circ} \mathrm{N}\right)$. The elevation and azimuth are easily computed from the formulae above. We plot the zenith angle (the complement of the elevation) and azimuth for the extra-tropical observation in Fig. 3. The observation point is at the centre, and the course of the Sun is shown by the curve. It is clear that the azimuth increases monotonically from sunrise to sunset, as we would expect.

In Fig. 4 we plot the zenith angle and azimuth for the observation point within the tropics $\left(\phi_{O}=20^{\circ} \mathrm{N}\right)$. At Noon, the Sun is to the North of the central point and the azimuthal angle is decreasing rapidly. This is the retrogression phenomenon. 


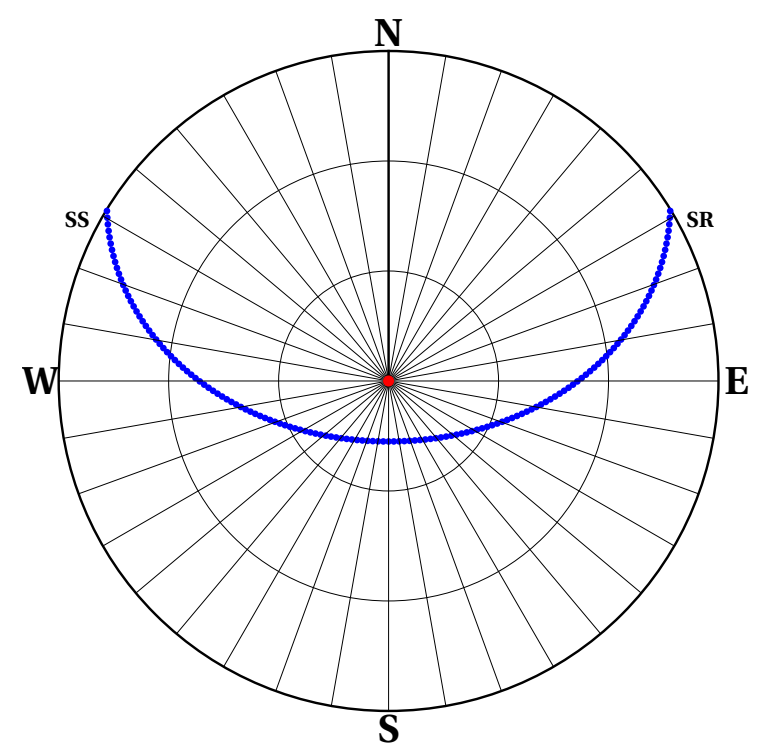

Figure 3. Path of the Sun at the Summer solstice for an observation point at $40^{\circ} \mathrm{N}$. The angular coordinate is the azimuth or compass bearing, $\alpha$. The radial coordinate is the zenith angle (the complement of the elevation). SR: Sunrise; SS: Sunset.

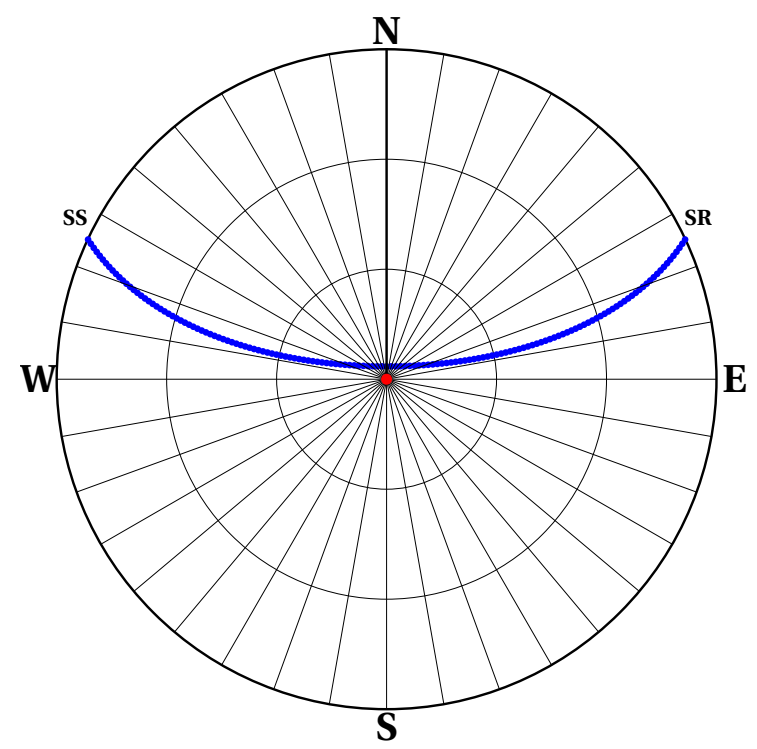

Figure 4. Path of the Sun at the Summer solstice for an observation point at $20^{\circ} \mathrm{N}$. The angular coordinate is the azimuth or compass bearing, $\alpha$. The radial coordinate is the zenith angle (the complement of the elevation). SR: Sunrise; SS: Sunset. 
The azimuth and elevation of the Sun are plotted in Fig. 5 for the two observation points. It is clear that when $\phi_{O}=40^{\circ}$ (top right panel) the azimuth increases monotonically, while when $\phi_{O}=20^{\circ}$ (bottom right panel) the azimuth increases from sunrise until about 10:00, then decreases until 14:00 and finally increases until sunset.
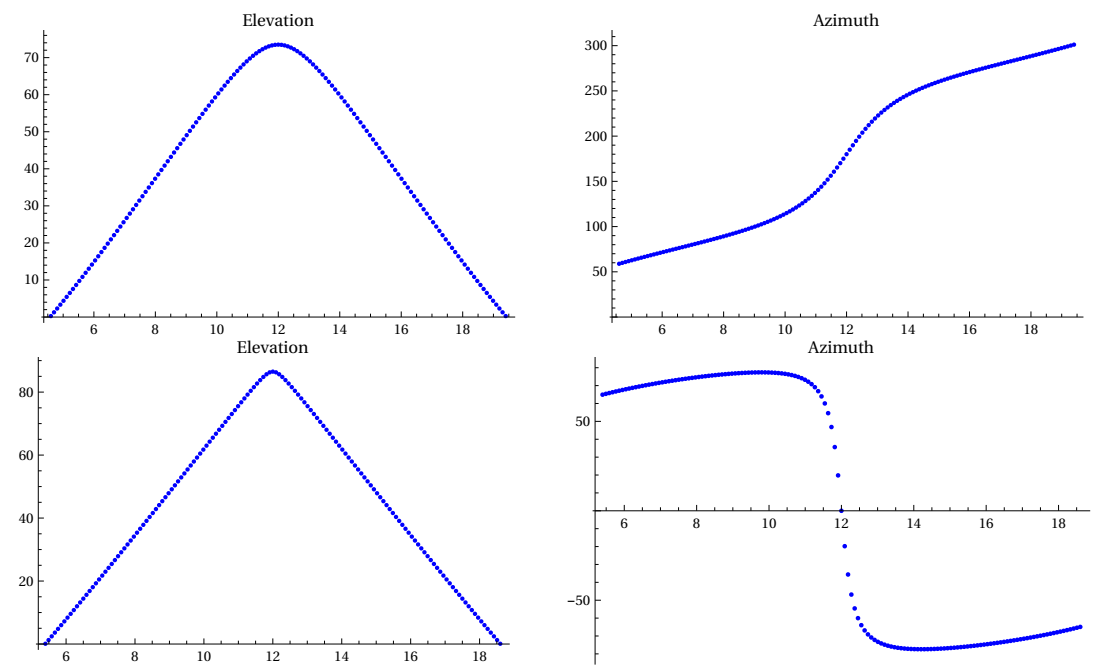

Figure 5. Solar elevation and azimuth, as functions of time from sunrise to sunset, for observation points at $40^{\circ} \mathrm{N}$ (top row) and $20^{\circ} \mathrm{N}$ (bottom row). Left: Solar elevation. Right: Solar azimuth.

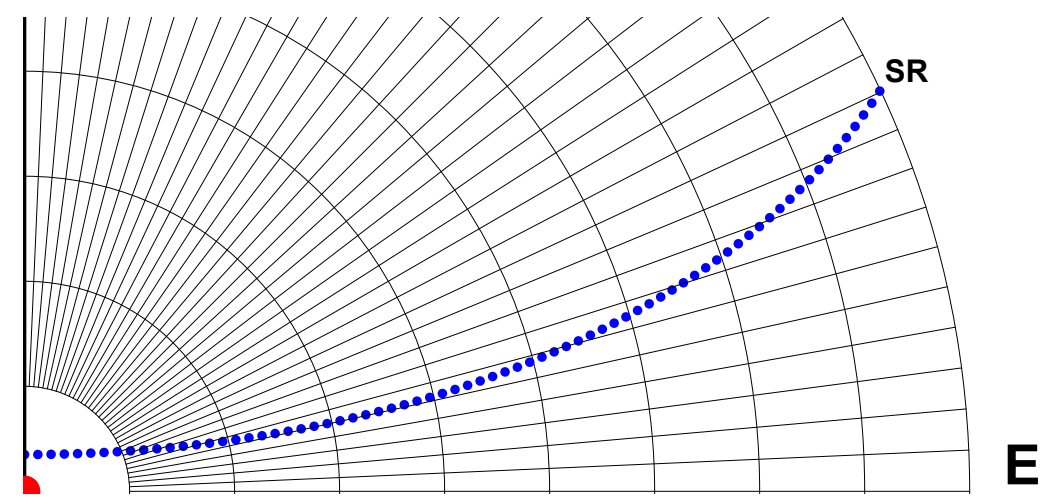

Figure 6. Path of the Sun at the Summer solstice for a observation point at $20^{\circ} \mathrm{N}$. Angular coordinate is the azimuth or compass bearing. Radial coordinate is the zenith angle. SR: Sunrise. Only the morning segment of the Sun's track is shown.

To give more fine detail, we plot the Sun's course during the morning, as seen from the tropical observatory, in Fig. 6. The radial lines 
are spaced five degrees apart, and we see that the azimuth at sunrise is close to $65^{\circ}$. It increases to around $77^{\circ}$ by mid-morning and then decreases to zero at Noon. For the specific values $\phi_{O}=20^{\circ}$ and $\phi_{S}=23.5^{\circ}$, the condition (5) gives the turning longitude as $\lambda_{S}=33.17^{\circ}$ corresponding to an azimuth of $77.4^{\circ}$ and an elevation of $59.1^{\circ}$. This is in excellent agreement with the numerical solution shown in Fig. 6 .

\section{REFERENCES}

[1] Leitão, Henrique, 2017: A brief note on the power of mathematics: Pedro Nunes and the retrogradation of shadows. Proceedings of Recreational Mathematics Colloquium V - G4G (Europe), Ed. Jorge Nuno Silva, pp. 45-52.

[2] Morrison, J, 1898: The sun dial of Ahaz. Popular Astronomy, 6 (10), 537-549.

[3] Nunes, Pedro, 1537: Treatise in defence of the nautical chart. For a modern edition see Obras de Pedro Nunes (Pedro Nunes's Complete Works), Lisbon Acad. Sci., Vol. I, 156-157.

Peter Lynch is Emeritus Professor in the School of Mathematics and Statistics, UCD. His interests include dynamic meteorology, numerical weather prediction and recreational mathematics. He writes an occasional mathematics column That's Maths in the Irish Times; see his blog at http://thatsmaths.com.

School of Mathematics and Statistics, University College Dublin E-mail address: Peter.Lynch@ucd.ie 